Brief paper

\title{
Static output-feedback stabilization of discrete-time Markovian jump linear systems: A system augmentation approach
}

\author{
Zhan Shu ${ }^{\mathrm{a}, \mathrm{b}}$, James Lam ${ }^{\mathrm{b}, *}$, Junlin Xiong ${ }^{\mathrm{c}}$ \\ ${ }^{a}$ Hamilton Institute, National University of Ireland, Maynooth, Maynooth, Co. Kildare, Ireland \\ ${ }^{\mathrm{b}}$ Department of Mechanical Engineering, The University of Hong Kong, Pokfulam Road, Hong Kong \\ ${ }^{\mathrm{c}}$ School of Engineering and Information Technology, University of New South Wales at the Australian Defence Force Academy, Canberra ACT 2600, Australia
}

\section{A R T I C L E I N F O}

\section{Article history:}

Received 16 October 2007

Received in revised form 17 August 2009

Accepted 3 January 2010

Available online 7 March 2010

\section{Keywords:}

Linear matrix inequality (LMI)

Markovian jump systems

Static output-feedback

System augmentation

Stabilization

\begin{abstract}
A B S T R A C T
This paper studies the static output-feedback (SOF) stabilization problem for discrete-time Markovian jump systems from a novel perspective. The closed-loop system is represented in a system augmentation form, in which input and gain-output matrices are separated. By virtue of the system augmentation, a novel necessary and sufficient condition for the existence of desired controllers is established in terms of a set of nonlinear matrix inequalities, which possess a monotonic structure for a linearized computation, and a convergent iteration algorithm is given to solve such inequalities. In addition, a special property of the feasible solutions enables one to further improve the solvability via a simple D-K type optimization on the initial values. An extension to mode-independent SOF stabilization is provided as well. Compared with some existing approaches to SOF synthesis, the proposed one has several advantages that make it specific for Markovian jump systems. The effectiveness and merit of the theoretical results are shown through some numerical examples.
\end{abstract}

(C) 2010 Elsevier Ltd. All rights reserved.

\section{Introduction}

Discrete-time Markovian jump linear systems (DMJLSs), modeled by a set of discrete-time linear systems with transitions among the models determined by a Markov chain taking values in a finite set, have appealed to a lot of researchers in the control community. This is partially due to their widespread applications to modeling various practical processes that experience abrupt changes in their structure and parameters, possibly caused by phenomena such as component failures or repairs, sudden environmental disturbances, and changing subsystem interconnections. Stability of DMJLSs has been investigated thoroughly in Costa and Fragoso (1993), and the equivalence of different second moment stability has been established in Ji, Chizeck, Feng, and Loparo (1991). The linear quadratic optimal control problem for DMJLSs has been studied in Chizeck, Willsky, and Castanon

\footnotetext{
This work was partially supported by RGC HKU 7029/05P and the Science Foundation of Ireland, Grant no. 07/PI/I1838. Part of the material in this article was presented at IFAC World Congress 2008. This paper was recommended for publication in revised form by Associate Editor Giuseppe De Nicolao under the direction of Editor Ian R. Petersen.

* Corresponding author. Tel.: +852 28592805; fax: +852 28592805, +852 28585415.

E-mail addresses: zhan.shu@nuim.ie (Z. Shu), james.lam@hku.hk (J. Lam), junlin.xiong@gmail.com (J. Xiong).
}

(1986) and Costa and de Paulo (2007), and the filtering problem has been considered in Costa and Marques (2000). Some results on the $\mathscr{H}_{2}$ and $\mathscr{H}_{\infty}$ control problems are available in Costa and Marques (1998), Seiler and Sengupta (2003) and references therein. Recent advances and applications related to networked control systems have been provided in Xiong and Lam (2007) and Huang and Dey (2007). As for robust stability analysis, we refer readers to de Souza (2006), Karan, Shi, and Kaya (2006) and references therein. More details on DMJLSs can be found in Costa, Fragoso, and Marques (2005).

In most of the literature, it is often assumed that the system state and mode are completely accessible to the controller. This assumption, however, may not be always true in practice, and it is necessary to consider the more practical case that the system state and mode are partially accessible. Our goal to seek an effective and easy-to-use approach to SOF control of DMJLSs is motivated not only by the fact that the system state and mode are not always accessible, but also by the simplicity of SOF to implement in practice. Moreover, many dynamic output-feedback synthesis problems can be reformulated as an SOF control design involving augmented plants. Although Costa, Do Val, and Geromel (1997) proposed a nonconvex cutting-plane algorithm based on the output structural constraint approach (Geromel, Peres, \& Souza, 1993 ) to solve the SOF $\mathscr{H}_{2}$ control problem for DMJLSs, there is still much room for improvement. The major obstacles in SOF synthesis of DMJLSs are not only the nonconvexity of the SOF problem 
itself, but also the coupled linear matrix inequality condition for stochastic stability of DMJLSs, which poses a severe problem in nonconservative controller synthesis (Lee \& Dullerud, 2006).

In this paper, we investigate the SOF stabilization problem for DMJLSs from a new point of view. The closed-loop system is represented in a system augmentation form with algebraic constraints. A new stability characterization of the closed-loop system is then established. Based on this new characterization, a necessary and sufficient condition with redundant matrix variables for mode-dependent SOF stabilizability is proposed, and an iteration algorithm is given to solve the condition. An extension to mode-independent SOF stabilization is provided as well. Several numerical examples are provided to show the effectiveness and merit of the theoretical results. In addition, the problems of $\mathscr{H}_{\infty}$ control, $\mathscr{H}_{2}$ control and mixed $\mathscr{H}_{2} / \mathscr{H}_{\infty}$ control with the SOF controllers can be readily treated under the framework (Shu, Lam, \& Xiong, 2008). Compared with some existing approaches to SOF synthesis, the proposed one has several advantages that make it specific for DMJLSs. First, the proposed approach does not employ any coordination transformation often used by other approaches, and thus can avoid any difficulty caused by multi-mode transformation matrices. Second, since the proposed approach does not use the Lyapunov matrices to parametrize controller gains and does not involve any controller reconstruction, it can cope with mode-independent control readily. In addition, the proposed approach may be numerically more desirable over those relying on the solution of Riccati equation due to the arduousness of solving coupled Riccati equations for DMJLSs.

Notation. Throughout this paper, for real symmetric matrices $X$ and $Y$, the notation $X>Y$ means that the matrix $X-Y$ is positivedefinite. $\|\cdot\|$ denotes the Euclidean norm for vectors and the spectral norm for matrices, respectively. $\mathcal{E}\{\cdot\}$ stands for the mathematical expectation with some probability measure. For a real matrix $C, C^{\perp}$ denotes an orthonormal basis of the null space of $C$, namely, $C C^{\perp}=0$ and $\left(C^{\perp}\right)^{T} C^{\perp}=I$. The symbol \# is used to denote a matrix which can be inferred by symmetry. Matrices, if their dimensions are not explicitly stated, are assumed to have compatible dimensions for algebraic operations.

\section{Preliminaries}

Consider the following class of discrete-time Markovian jump systems:

$$
\left\{\begin{array}{l}
x(k+1)=A(r(k)) x(k)+B(r(k)) u(k), \\
y(k)=C(r(k)) x(k),
\end{array}\right.
$$

where $x(k) \in \mathbb{R}^{n}, u(k) \in \mathbb{R}^{l}$, and $y(k) \in \mathbb{R}^{m}$ are the system state, the control input, and the measured output, respectively, and $A(r(k)), B(r(k))$, and $C(r(k))$ are the system matrices of the stochastic jumping process $\{r(k), k \geq 0\}$; the parameter $r(k)$ represents a discrete-time, discrete-state Markov chain taking values in a finite set $s=\{1,2, \ldots, s\}$ with transition probabilities $\pi_{i j}, \pi_{i j} \geq 0$ and $\sum_{j=1}^{s} \pi_{i j}=1, \forall i \in \&$. To simplify the notation, $M(r(k))$ and $M_{N}(r(k))$ will be denoted by $M_{r(k)}$ and $M_{N r(k)}$, respectively, and, for a set of matrices $M_{i}, i \in \delta$,

$\hat{M}_{i} \triangleq \sum_{j=1}^{s} \pi_{i j} M_{j}$

Definition 1. The system in (1) is said to be stochastically stable if, when $u(k) \equiv 0$, there exists a scalar $\tilde{M}\left(x_{0}, r_{0}\right)>0$ such that

$\lim _{\nu \rightarrow \infty} \varepsilon\left\{\sum_{k=0}^{v}\|x(k)\|^{2} \mid x_{0}, r_{0}\right\} \leq \tilde{M}\left(x_{0}, r_{0}\right)$.
The SOF controller under consideration is of the form $u(k)=$ $K_{r(k)} y(k)$. Connecting this controller to (1) yields the closed-loop system

$x(k+1)=A_{c l r}(k) x(k)$,

where $A_{c l r(k)}=A_{r(k)}+B_{r(k)} K_{r(k)} C_{r(k)}$. Our goal is to design $K_{i}, \forall i \in \&$, such that the system in (2) is stochastically stable. Since $K_{r(k)}$ is embedded in the middle of two matrices, it is hard to parametrize it by matrix variables. Hence, our fundamental idea is to extract $K_{r(k)}$ from the middle of two matrices. To this end, we view the input $u(k)$ as a state component and choose $\left[\begin{array}{ll}x^{T}(k) & u^{T}(k)\end{array}\right]^{T}$ as the new state variable, and rewrite the closed-loop system as follows:

$\mathbf{E} \xi(k+1)=\mathbf{A}_{r(k)} \xi(k)$,

where $\xi(k)=\left[\begin{array}{ll}x^{T}(k) & u^{T}(k)\end{array}\right]^{T}, \mathbf{E}=\left[\begin{array}{ll}I & 0 \\ 0 & 0\end{array}\right]$, and $\mathbf{A}_{r(k)}=$ $\left[\begin{array}{cc}A_{r(k)} & B_{r(k)} \\ K_{r(k)} C_{r(k)} & -I\end{array}\right]$.

Remark 1. An advantage of the system augmentation lies in the separation of $B_{r(k)}$ and $K_{r(k)} C_{r(k)}$, which enables us to parametrize $K_{r(k)}$ by free parameter matrices. It is noted that if we choose $\left[\begin{array}{ll}x^{T}(t) & y^{T}(t)\end{array}\right]^{T}$ as a new state variable, we can also obtain a similar system augmentation, which we call dual system augmentation. In this paper, we do not intend to present any results on dual system augmentation, due to the page length consideration, and further discussion on this issue will appear in our future work. In addition, many dynamic output-feedback synthesis problems can be reformulated as an SOF control design involving augmented plants (Syrmos, Abdallah, Dorato, \& Grigoriadis, 1997), and thus the approach presented in this paper is applicable to the dynamic output-feedback case as well.

\section{Closed-loop stability condition and equivalent characteriza- tion}

Theorem 1. The system in (2) is stochastically stable if and only if there exist matrices $P_{1 i}=P_{1 i}^{T}, P_{4 i}=P_{4 i}^{T}, P_{2 i}, Q_{4 i}>0$, and a scalar $\alpha>0$ such that, $\forall i \in \&$,

$\mathbf{A}_{i}^{T} \hat{\mathbf{P}}_{i} \mathbf{A}_{i}-\mathbf{E} \mathbf{P}_{i} \mathbf{E}+\mathbf{Q}_{i} \mathbf{A}_{i}+\mathbf{A}_{i}^{T} \mathbf{Q}_{i}^{T}<0$,

where

$\mathbf{P}_{i}=\left[\begin{array}{ll}P_{1 i} & P_{2 i}^{T} \\ P_{2 i} & P_{4 i}\end{array}\right]>0, \quad \mathbf{Q}_{i}=\left[\begin{array}{cc}0 & -\alpha C_{i}^{T} K_{i}^{T} Q_{4 i} \\ 0 & \alpha Q_{4 i}\end{array}\right]$.

Proof. (Sufficiency) Note that $\mathbf{A}_{i}$ has the following decomposition:

$\mathbf{A}_{i}=\left[\begin{array}{cc}A_{\text {cli }} & B_{i} \\ 0 & -I\end{array}\right]\left[\begin{array}{cc}I & 0 \\ -K_{i} C_{i} & I\end{array}\right]$.

With this and algebraic manipulations, one has that

$$
\begin{aligned}
\mathbf{A}_{i}^{T} \hat{\mathbf{P}}_{i} \mathbf{A}_{i} & -\mathbf{E P}_{i} \mathbf{E}+\mathbf{Q}_{i} \mathbf{A}_{i}+\mathbf{A}_{i}^{T} \mathbf{Q}_{i}^{T}=\left[\begin{array}{cc}
I & 0 \\
-K_{i} C_{i} & I
\end{array}\right]^{T} \\
\times & {\left[\begin{array}{cc}
A_{c l i}^{T} \hat{P}_{1 i} A_{c l i}-P_{1 i} & \# \\
B_{i}^{T} \hat{P}_{1 i} A_{c l i}-\hat{P}_{2 i} A_{c l i} & \left(\begin{array}{c}
B_{i}^{T} \hat{P}_{1 i} B_{i} \\
-\hat{P}_{2 i} B_{i}-B_{i}^{T} \hat{P}_{2 i}^{T} \\
+\hat{P}_{4 i}-2 \alpha Q_{4 i}
\end{array}\right)
\end{array}\right]\left[\begin{array}{cc}
I & 0 \\
-K_{i} C_{i} & I
\end{array}\right] . }
\end{aligned}
$$

This together with (4) yields that $A_{c l i}^{T} \hat{P}_{1 i} A_{c l i}-P_{1 i}<0$, which implies that the system in (2) is stochastically stable (Costa \& Fragoso, 1993; Ji et al., 1991). 
(Necessity) If the system in (2) is stochastically stable, then, according to the second moment stability criterion, there exist matrices $P_{1 i}>0, i \in \S$, such that $A_{c l i}^{T} \hat{P}_{1 i} A_{c l i}-P_{1 i}<0$. Now define matrices $\mathbf{P}_{i}$ and $\mathbf{Q}_{i}$ as

$\mathbf{P}_{i}=\left[\begin{array}{cc}P_{1 i} & 0 \\ 0 & P_{4 i}\end{array}\right], \quad \mathbf{Q}_{i}=\left[\begin{array}{cc}0 & -\alpha C_{i}^{T} K_{i}^{T} \hat{P}_{4 i} \\ 0 & \alpha \hat{P}_{4 i}\end{array}\right]$,

where $P_{4 i}, \forall i \in \S$, is any positive-definite matrix, and $\alpha>0$ is a sufficiently large scalar such that, $\forall i \in \&$,

$$
\begin{gathered}
B_{i}^{T} \hat{P}_{1 i} A_{c l i}\left(P_{1 i}-A_{c l i}^{T} \hat{P}_{1 i} A_{c l i}\right)^{-1} A_{c l i}^{T} \hat{P}_{1 i} B_{i} \\
+B_{i}^{T} \hat{P}_{1 i} B_{i}+(1-2 \alpha) \hat{P}_{4 i}<0 .
\end{gathered}
$$

Then, directly manipulating together with (7), (9), and using Schur complement equivalence yield that (4) holds with $P_{1 i}=P_{1 i}^{T}, P_{4 i}=$ $P_{4 i}^{T}, P_{2 i}=0, Q_{4 i}=\hat{P}_{4 i}$, and a sufficiently large scalar $\alpha>0$.

Remark 2. Since only $-\alpha Q_{4 i}$ are required to be negative definite, another choice is to let $\alpha<0$ and $Q_{4 i}<0$, and a corresponding necessary and sufficient condition can be similarly derived. In fact, it is easy to show that the case of $\alpha<0$ and $Q_{4 i}<0$ is equivalent to the case of $\alpha>0$ and $Q_{4 i}>0$. For simplicity, we only present the results for $\alpha>0$ and $Q_{4 i}>0$ throughout the paper.

In the following theorem, we provide an equivalent characterization on stability of the closed-loop system, which will play a key role in the subsequent controller synthesis.

Theorem 2. The system in (2) is stochastically stable if and only if there exist matrices $P_{1 i}=P_{1 i}^{T}, P_{4 i}=P_{4 i}^{T}, P_{2 i}, Q_{4 i}>0, H_{1 i}, H_{2 i}, G_{1 i}, G_{2 i}$, and a scalar $\alpha>0$ such that, $\forall i \in \delta$,

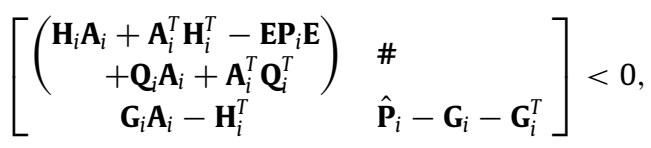

where $\mathbf{P}_{i}>0$ and $\mathbf{Q}_{i}$ are defined in Theorem 1, and

$$
\mathbf{H}_{i}=\left[\begin{array}{cc}
H_{1 i} & 0 \\
H_{2 i} & 0
\end{array}\right], \quad \mathbf{G}_{i}=\left[\begin{array}{cc}
G_{1 i} & 0 \\
G_{2 i} & Q_{4 i}
\end{array}\right] .
$$

Proof. (Sufficiency) Pre- and post-multiplying (10) by $\left[\begin{array}{ll}I & \mathbf{A}_{i}^{T}\end{array}\right]$ and its transpose yields that (4) holds.

(Necessity) Assume that the system in (2) is stochastically stable, then, according to Theorem 1 and its proof, there exist matrices

$\mathbf{P}_{i}=\left[\begin{array}{cc}P_{1 i} & 0 \\ 0 & P_{4 i}\end{array}\right]>0, \quad \mathbf{Q}_{i}=\left[\begin{array}{cc}0 & -\alpha C_{i}^{T} K_{i}^{T} Q_{4 i} \\ 0 & \alpha Q_{4 i}\end{array}\right]$,

with $\alpha>0$ and $Q_{4 i}=\hat{P}_{4 i}>0$ being a large enough scalar and positive definite matrices, respectively, such that $\mathbf{A}_{i}^{T} \hat{\mathbf{P}}_{i} \mathbf{A}_{i}-\mathbf{E P}_{i}$ $\mathbf{E}+\mathbf{Q}_{i} \mathbf{A}_{i}+\mathbf{A}_{i}^{T} \mathbf{Q}_{i}^{T}<0$, which, by Schur complement equivalence, implies

$$
\left[\begin{array}{cc}
-\mathbf{E} \mathbf{P}_{i} \mathbf{E}+\mathbf{Q}_{i} \mathbf{A}_{i}+\mathbf{A}_{i}^{T} \mathbf{Q}_{i}^{T} & \mathbf{A}_{i}^{T} \hat{\mathbf{P}}_{i} \\
\hat{\mathbf{P}}_{i} \mathbf{A}_{i} & -\hat{\mathbf{P}}_{i}
\end{array}\right]<0 .
$$

Now define $\mathbf{H}_{i}=\left[\begin{array}{ll}0 & 0 \\ 0 & 0\end{array}\right], \mathbf{G}_{i}=\left[\begin{array}{cc}\hat{P}_{1 i} & 0 \\ 0 & Q_{4 i}\end{array}\right]$.Then, we obtain that (10) holds with $P_{1 i}=P_{1 i}^{T}, P_{4 i}=P_{4 i}^{T}, P_{2 i}=0, Q_{4 i}=\hat{P}_{4 i}, H_{1 i}=0$, $H_{2 i}=0, G_{1 i}=\hat{P}_{1 i}, G_{2 i}=0$, and a sufficiently large scalar $\alpha>0$. This completes the proof.
Remark 3. In most existing LMI formulations, the multiplication of the Lyapunov matrices and the controller matrices may induce additional constraints on the Lyapunov matrices when the controller matrices are parametrized, and thus makes the corresponding results conservative. The significance of the condition in (10) lies not only in the separation of $B_{i}$ and $K_{i} C_{i}$, but also in the separation of the Lyapunov matrices $P_{1 i}$ and the controller matrices $K_{i}$, which avoids imposing any constraint on $P_{1 i}$ when $K_{i}$ is parametrized. In addition, redundant matrix variables $H_{1 i}, H_{2 i}, G_{1 i}$, and $G_{2 i}$, which are expected to reduce the conservatism and to improve the solvability of the iterative calculation to be presented later, are introduced by following the idea proposed in de Oliveira, Bernussou, and Geromel (1999).

Remark 4. One may argue that the idea to introduce slack matrices (or multipliers) proposed in de Oliveira et al. (1999) has already been used to solve the control and filter problems for MJLSs or the usual discrete-time systems (do Val, Geromel, \& Goncalves, 2002; Du, Xie, Teoh, \& Guo, 2005; Gao, Lam, Xie, \& Wang, 2005; K.H. Lee, J.H. Lee, \& Kwon, 2006). However, our characterization has essential differences from theirs. On one hand, the matrix coupled with the controller matrix in do Val et al. (2002); Du et al. (2005) and Gao et al. (2005); Lee et al. (2006) has to be equal (or related) to the Lyapunov matrix when the necessity needs to be proved, whereas the parametrization matrix $Q_{4 i}$ in (10) has nothing to do with the Lyapunov matrix. This can avoid introducing the conservatism when additional design specifications are involved. On the other hand, without coordinate transformation or additional constraints, their approaches may not parametrize the controller matrices, whereas, based on the system augmentation, (10) can directly as revealed later.

\section{Controller synthesis}

We present a necessary and sufficient condition for modedependent SOF stabilizability in the following theorem.

Theorem 3. The system in (1) is SOF stabilizable by a modedependent controller if and only if there exist a scalar $\alpha>0$, and matrices $P_{1 i}=P_{1 i}^{T}, P_{4 i}=P_{4 i}^{T}, P_{2 i}, H_{1 i}, H_{2 i}, G_{1 i}, G_{2 i}, Q_{4 i}>0, L_{i}, M_{i}$, such that, $\forall i \in$ \&,

$\left[\begin{array}{ll}P_{1 i} & P_{2 i}^{T} \\ P_{2 i} & P_{4 i}\end{array}\right]>0$

$\Omega_{i}\left(\alpha, M_{i}\right) \triangleq\left[\begin{array}{cccc}\Omega_{11 i} & \# & \# & \# \\ \Omega_{21 i} & \Omega_{22 i} & \# & \# \\ \Omega_{31 i} & \Omega_{32 i} & \Omega_{33 i} & \# \\ \Omega_{41 i i} & \Omega_{42 i} & \Omega_{43 i} & \Omega_{44 i}\end{array}\right]<0$,

where

$$
\begin{aligned}
\Omega_{11 i}= & H_{1 i} A_{i}+A_{i}^{T} H_{1 i}^{T}-P_{1 i} \\
& +2 \alpha M_{i}^{T} Q_{4 i} M_{i}-2 \alpha C_{i}^{T} L_{i}^{T} M_{i}-2 \alpha M_{i}^{T} L_{i} C_{i}, \\
\Omega_{21 i}= & H_{2 i} A_{i}+B_{i}^{T} H_{1 i}^{T}+2 \alpha L_{i} C_{i}, \\
\Omega_{22 i}= & H_{2 i} B_{i}+B_{i}^{T} H_{2 i}^{T}-2 \alpha Q_{4 i}, \\
\Omega_{31 i}= & G_{1 i} A_{i}-H_{1 i}^{T}, \\
\Omega_{32 i}= & G_{1 i} B_{i}-H_{2 i}^{T}, \\
\Omega_{33 i}= & \hat{P}_{1 i}-G_{1 i}-G_{1 i}^{T}, \\
\Omega_{41 i}= & G_{2 i} A_{i}+L_{i} C_{i}, \\
\Omega_{42 i}= & G_{2 i} B_{i}-Q_{4 i}, \\
\Omega_{43 i}= & -G_{2 i}+\hat{P}_{2 i}, \\
\Omega_{44 i}= & \hat{P}_{4 i}-2 Q_{4 i} .
\end{aligned}
$$


Under the conditions, an SOF control law can be obtained as

$K_{i}=Q_{4 i}^{-1} L_{i}$.

Proof. (Sufficiency) Since $Q_{4 i}>0,(15)$ is meaningful and $L_{i}=$ $Q_{4 i} K_{i}$. Substituting this into (14) and noticing that

$$
\begin{aligned}
-2 \alpha C_{i}^{T} K_{i}^{T} Q_{4 i} K_{i} C_{i} \leq & -2 \alpha\left(C_{i}^{T} K_{i}^{T} Q_{4 i}^{T}\right) M_{i}-2 \alpha M_{i}^{T}\left(Q_{4 i} K_{i} C_{i}\right) \\
& +2 \alpha M_{i}^{T} Q_{4 i} M_{i},
\end{aligned}
$$

we obtain that (10) holds. This proves the sufficiency.

(Necessity) Assume that (10) holds. Then, by choosing $M_{i}=$ $K_{i} C_{i}$, we have that $-2 \alpha C_{i}^{T} K_{i}^{T} Q_{4 i} K_{i} C_{i}=-2 \alpha\left(C_{i}^{T} K_{i}^{T} Q_{4 i}^{T}\right) M_{i}-2 \alpha M_{i}^{T}$ $\left(Q_{4 i} K_{i} C_{i}\right)+2 \alpha M_{i}^{T} Q_{4 i} M_{i}$. Substituting this into (10) and letting $L_{i}=$ $Q_{4 i} K_{i}$, we obtain that (14) holds. This completes the proof.

Remark 5. It is noted that, without loss of generality, the matrices $P_{4 i}, Q_{4 i}$, and $L_{i}$ in Theorem 3 can be set to be mode-independent, that is, $P_{41}=P_{42}=\cdots=P_{4 s}, Q_{41}=Q_{42}=\cdots=Q_{4 s}, L_{1}=$ $L_{2}=\cdots=L_{s}$, and the corresponding stability conditions are still necessary and sufficient. In view of this feature, it is easy to design a mode-independent controller for the case that the jump variable $r(k)$ is not available without imposing any restriction on the Lyapunov matrices $P_{1 i}$, which may cause excessive conservatism. Indeed, the parametrization of the controller matrices by our approach is fairly flexible, and $P_{4}$ and $Q_{4}$ can be further set to be the identity matrix while not causing loss of generality. In this case, many synthesis problems, such as simultaneous stabilization and structural controller synthesis, can be treated readily under the same framework.

When $\alpha$ and $M_{i}$ are fixed, (14) becomes a strict LMI for each mode, which could be verified easily by conventional LMI solver. According to the previous analysis, the larger the $\alpha$, the higher the reduction in conservatism of (14). If (14) does not hold for a sufficiently large $\alpha>0$, it is plausible to conclude that the system is not SOF stabilizable. It is not difficult to show that, when $M_{i}=Q_{4 i}^{-1} L_{i} C_{i}$, the left side of (14) is a monotonic decreasing matrix function with respect to $\alpha$. Hence, we can set $\alpha$ to be large values. The remaining problem is how to select $M_{i}$. It can be seen from the proof of Theorem 3 that the scalar $\gamma$ satisfying $\Omega_{i}\left(\alpha, M_{i}\right)<\gamma I$ achieves its minimum when $M_{i}=Q_{4 i}^{-1} L_{i} C_{i}$, which can be used to construct an iteration rule. We summarize briefly our analysis on $\alpha$ and $M_{i}$ in the following proposition.

Proposition 1. When $P_{1 i}>0, P_{4 i}>0, P_{2 i}, H_{1 i}, H_{2 i}, G_{1 i}, G_{2 i}, Q_{4 i}>0$, and $L_{i}$ are fixed, the following relationship holds for any $M_{i}$ and $\alpha_{1}>$ $\alpha_{2}>0$,

$\Omega_{i}\left(\alpha_{1}, Q_{4 i}^{-1} L_{i} C_{i}\right) \leq \Omega_{i}\left(\alpha_{2}, Q_{4 i}^{-1} L_{i} C_{i}\right) \leq \Omega_{i}\left(\alpha_{2}, M_{i}\right)$.

Proof. The second " $\leq$ " follows immediately from the proof of Theorem 3. As for the first " $\leq$ ", direct algebraic operations give that $\Omega_{i}\left(\alpha_{1}, Q_{4 i}^{-1} L_{i} C_{i}\right)-\Omega_{i}\left(\alpha_{2}, Q_{4 i}^{-1} L_{i} C_{i}\right)$

$$
=2\left(\alpha_{2}-\alpha_{1}\right)\left[\begin{array}{c}
-C_{i}^{T} L_{i}^{T} \\
Q_{4 i} \\
0 \\
0
\end{array}\right] Q_{4 i}^{-1}\left[\begin{array}{c}
-C_{i}^{T} L_{i}^{T} \\
Q_{4 i} \\
0 \\
0
\end{array}\right]^{T}
$$

$\leq 0$

Therefore, the following iteration algorithm is constructed to solve the condition of Theorem 3 .

\section{Algorithm 1.}

1. Set $v=1$ and $\alpha$ to be a sufficiently large value (for example, $\left.\alpha=10^{4}\right)$. Select initial values $M_{i}^{(v)}$ such that $x(k+1)=$
$\left[A_{r(k)}+B_{r(k)} M_{r(k)}^{(v)}\right] x(k)$ is stochastically stable.

2. For fixed $\alpha$ and $M_{i}^{(v)}$, minimize $\gamma^{(v)}$ subject to (13), $Q_{4 i}^{(v)}>0$, and

$\Omega_{i}\left(\alpha, M_{i}^{(v)}\right)<\gamma^{(v)} I$,

$\gamma^{(v)} \geq-c$

where $\Omega_{i}\left(\alpha, M_{i}^{(v)}\right)$ is defined in (14), and $c$ is any prescribed positive real number. If a $\gamma_{l t z}^{(v)} \leq 0$ is found during solving the convex optimization problem, then the system is SOF stabilizable, and a control law can be obtained as (15). STOP.

3. Denote $\gamma_{*}^{(v)}$ as the optimal value of $\gamma^{(v)}$. If $\left|\gamma_{*}^{(v)}-\gamma_{*}^{(v-1)}\right| \leq \delta$, where $\delta$ is a prescribed tolerance, then goto next step, else update $M_{i}^{(v+1)}$ as

$M_{i}^{(v+1)}=\left(Q_{4 i}^{(v)}\right)^{-1} L_{i}^{(v)} C_{i}$,

and set $v=v+1$, then goto step 2 .

4. The system may not be SOF stabilizable. STOP (or choose larger $\alpha$ and other initial values $M_{i}^{(1)}$, then run the algorithm again).

Remark 6. The sequence $\gamma_{*}^{(v)}$ is monotonic decreasing with respect to $v$, that is, $\gamma_{*}^{(v)} \leq \gamma_{*}^{(v-1)}$, and lower bounded from $-c$, and thus the convergence of the iteration is guaranteed.

Remark 7. The initial values $M_{i}^{(1)}$ are the state-feedback stabilizing controller matrices, which can be found by existing approaches (Costa et al., 1997; Ji et al., 1991). If no such matrices are found, we can conclude immediately that the system is not SOF stabilizable. Like many other iterative algorithms (Cao, Lam, \& Sun, 1998; Cao, Sun, \& Mao, 1998; Fujimori, 2004; Gadewadikar, Lewis, Xie, Kucera, \& Abu-Khalaf, 2007; Iwasaki, 1999), the sequence of iterates depends on the selection of initial values, and appropriate selection of $M_{i}^{(1)}$ will improve the solvability. In addition, it should be emphasized that the tuning parameter $\alpha$ may affect the optimum of the converged value $\gamma_{*}^{(\infty)}$, although larger $\alpha$ make the condition less stringent. More specifically, for $\alpha_{1}>\alpha_{2}$, it is possible that $\gamma_{* 1}^{(\infty)} \geq \gamma_{* 2}^{(\infty)}$, which means that $\gamma$ may not always converge to its global minimum. According to the authors' numerical experience, $\alpha$ should be chosen between $10^{3}$ and $10^{6}$ for most cases.

Remark 8. For each iteration, $\Omega_{i}\left(\alpha, M_{i}^{(\nu)}\right)<0$ is no longer necessary for the existence of the desired controllers, since $\alpha$ and $M_{i}^{(v)}$ are fixed. Therefore, it is expected that $H_{1 i}, H_{2 i}, G_{1 i}$, and $G_{2 i}$ are helpful for finding a lower $\gamma^{(v)}$.

Remark 9. Due to the separation of the Lyapunov matrices and the system matrices, the results can be extended to polytopic uncertain model with parameter-dependent Lyapunov matrices in a straightforward manner. If one is only concerned with the model without uncertainties, one may eliminate $H_{1 i}, H_{2 i}, G_{1 i}$, and $G_{2 i}$ via the nonconservative projection (Boyd, El Ghaoui, Feron, \& Balakrishnan, 1994) to reduce computation burdens.

Based on the proposed approach, the problems of $\mathscr{H}_{\infty}$ control, $\mathscr{H}_{2}$ control and mixed $\mathscr{H}_{2} / \mathscr{H}_{\infty}$ control with the SOF controllers can be readily treated under the same framework. Further results on these topics are available in Shu et al. (2008).

By setting $Q_{4 i}$ to be mode-independent, as stated in Remark 5, and following the same derivation, we establish a necessary and sufficient condition for mode-independent SOF stabilizability in the following theorem.

Theorem 4. The system in (1) is SOF stabilizable by a modeindependent controller if and only if there exist a scalar $\alpha>0$, and matrices $P_{1 i}=P_{1 i}^{T}, P_{4 i}=P_{4}>0, P_{2 i}, H_{1 i}, H_{2 i}, G_{1 i}, G_{2 i}, Q_{4 i}=Q_{4}>0$, $L_{i}=L, M_{i}$, such that, $\forall i \in \S,(13)$ and (14) hold. Under the conditions, an SOF control law can be obtained as $K=Q_{4}^{-1} L$. 
An algorithm similar to the mode-dependent case (Algorithm 1) can be constructed, but omitted here for brevity.

\section{Optimization of initial values}

As mentioned in Remark 7, the initial values $M_{i}$ and the tuning parameter $\alpha$ may affect the optimum of the iteration. To see this in a detailed way, let us consider (16), namely,

$$
\begin{aligned}
-2 \alpha C_{i}^{T} K_{i}^{T} Q_{4 i} K_{i} C_{i} \leq & -2 \alpha C_{i}^{T} K_{i}^{T} Q_{4 i} K_{i} C_{i} \\
& +2 \alpha\left(M_{i}-K_{i} C_{i}\right)^{T} Q_{4 i}\left(M_{i}-K_{i} C_{i}\right) .
\end{aligned}
$$

It follows from this inequality that desired $\alpha$ and $M_{i}^{(1)}$ should make $\left\|2 \alpha\left(M_{i}-K_{i} C_{y i}\right)^{T} Q_{4 i}\left(M_{i}-K_{i} C_{y i}\right)\right\|$ small. The reduction of $\alpha$, however, contradicts with the requirement of $\alpha$ being sufficiently large, which makes the condition less stringent. Hence, the only way is to reduce $\left\|M_{i}^{(1)}-K_{i} C_{i}\right\|$ by choosing appropriate $M_{i}^{(1)}$. To this end, we provide the following proposition, which plays a central role in selecting $M_{i}^{(1)}$.

Proposition 2. For the system in (1) and matrices $F_{i}$, the following statements are equivalent.

(I) There exist $K_{i}^{*}, \forall i \in$ \&, such that (2) is stochastically stable, and $\left\|F_{i}-K_{i}^{*} C_{i}\right\| \leq \epsilon_{1}$, where $\epsilon_{1}>0$ is a sufficiently small scalar.

(II) $x(k+1)=\left(A_{r(k)}+B_{r(k)} F_{r(k)}\right) x(k)$ is stochastically stable and $\left\|F_{i} C_{i}^{\perp}\right\| \leq \epsilon_{2}$, where $\epsilon_{2}>0$ is a sufficiently small scalar.

Proof. ((I) $\Rightarrow$ (II)) It follows from (I) that

$\left\|\left(A_{i}+B_{i} F_{i}\right)-\left(A_{i}+B_{i} K_{i}^{*} C_{i}\right)\right\|=\left\|B_{i}\left(F_{i}-K_{i}^{*} C_{i}\right)\right\|$

is sufficiently small, and stochastic stability of $x(k+1)=$ $\left(A_{r(k)}+B_{r(k)} F_{r(k)}\right) x(k)$ can be inferred from this and stability analysis provided in Costa and Fragoso (1993). In addition, we have that

$\left\|F_{i} C_{i}^{\perp}\right\|=\left\|F_{i} C_{i}^{\perp}-K_{i}^{*} C_{i} C_{i}^{\perp}\right\| \leq \epsilon_{1} \triangleq \epsilon_{2}$.

$((\mathrm{II}) \Rightarrow(\mathrm{I}))$ It is noted that if $\operatorname{rank}\left(C_{i}\right)=m_{i}<m$, then $C_{i}$ can be QR-factorized as $C_{i}=U_{i}\left[\begin{array}{ll}C_{1 i}^{T} & 0\end{array}\right]^{T}$, where $U_{i} \in \mathbb{R}^{m \times m}$ is an orthogonal matrix, and $C_{1 i} \in \mathbb{R}^{m_{i} \times n}$ is a matrix with full row rank. Now define $K_{i}^{*}$ as



which implies that $F_{i} C_{i}^{T}-K_{i}^{*} C_{i} C_{i}^{T}=0$, when $\operatorname{rank}\left(C_{i}\right)=m$, and $F_{i} C_{1 i}^{T}-K_{i}^{*} C_{i} C_{1 i}^{T}=0$, when $\operatorname{rank}\left(C_{i}\right)<m$. By this and noting $C_{1 i}^{\perp}=C_{i}^{\perp}$, we obtain that

$\left(F_{i}-K_{i}^{*} C_{i}\right)\left[\begin{array}{ll}C_{i}^{T} & C_{i}^{\perp}\end{array}\right]=\left[\begin{array}{ll}0 & F_{i} C_{i}^{\perp}\end{array}\right], \quad \operatorname{rank}\left(C_{i}\right)=m$,

$\left(F_{i}-K_{i}^{*} C_{i}\right)\left[\begin{array}{ll}C_{1 i}^{T} & C_{1 i}^{\perp}\end{array}\right]=\left[\begin{array}{ll}0 & F_{i} C_{i}^{\perp}\end{array}\right], \quad \operatorname{rank}\left(C_{i}\right)<m$,

which, together with the invertibility of $\left[\begin{array}{ll}C_{i}^{T} & C_{i}^{\perp}\end{array}\right]$ and $\left[\begin{array}{ll}C_{1 i}^{T} & C_{1 i}^{\perp}\end{array}\right]$, infers to

$$
\begin{aligned}
\left\|F_{i}-K_{i}^{*} C_{i}\right\| & \leq \epsilon_{2} \max _{i \in s}\left\{\max \left\{\left\|\left[\begin{array}{ll}
C_{i}^{T} & C_{i}^{\perp}
\end{array}\right]^{-1}\right\|,\left\|\left[\begin{array}{ll}
C_{1 i}^{T} & C_{1 i}^{\perp}
\end{array}\right]^{-1}\right\|\right\}\right\} \\
& \triangleq \epsilon_{1} .
\end{aligned}
$$

Similar to the derivation in ((I) $\Rightarrow$ (II)), we further obtain that $x(k+1)=\left(A_{i}+B_{i} K_{i}^{*} C_{i}\right) x(k)$ is stochastically stable.

On the basis of this proposition, a D-K type optimization algorithm is provided to find appropriate initial values $M_{i}^{(1)}$.

\section{Algorithm 2.}

1. Set $v=1$, and select matrices $F_{i}^{(1)}, i \in \S$, such that $x(k+1)=$ $\left(A_{r(k)}+B_{r(k)} F_{r(k)}^{(1)}\right) x(k)$ is stochastically stable.
2. For fixed $F_{i}^{(v)}$, find $P_{i}^{(v)}$ such that

$$
\left(A_{i}+B_{i} F_{i}^{(v)}\right)^{T} \hat{P}_{i}^{(v)}\left(A_{i}+B_{i} F_{i}^{(v)}\right)-P_{i}^{(v)}<0 .
$$

3. For fixed $P_{i}^{(v)}$, minimize $\epsilon^{(v)}$ subject to

$$
\begin{aligned}
& {\left[\begin{array}{cc}
-\epsilon^{(v)} I & \# \\
F_{i}^{(v)} C_{i}^{\perp} & -I
\end{array}\right]<0,} \\
& {\left[\begin{array}{cc}
-P_{i}^{(v)} & \left(A_{i}+B_{i} F_{i}^{(v)}\right)^{T} \hat{P}_{i}^{(v)} \\
\hat{P}_{i}^{(v)}\left(A_{i}+B_{i} F_{i}^{(v)}\right) & -\hat{P}_{i}^{(v)}
\end{array}\right]<0 .}
\end{aligned}
$$

4. Denote $\epsilon_{*}^{(v)}$ and $F_{i *}^{(v)}$ as the optimal value of $\epsilon^{(v)}$ and $F_{i}^{(v)}$. If $\epsilon_{*}^{(v)} \leq \delta_{1}$, a prescribed tolerance, then desirable initial values $M_{i}^{(1)}=F_{i *}^{(v)}$ can be found. STOP.

5. If $\left|\epsilon_{*}^{(\nu)}-\epsilon_{*}^{(\nu-1)}\right| \leq \delta_{2}$, a prescribed tolerance, then goto next step, else set $v=v+1, F_{i}^{(v)}=F_{i *}^{(v-1)}$, and goto step 2 .

6. Desired initial values cannot be found. STOP.

It can be shown readily that $\epsilon^{(v)}$ is decreasing with respect to $v$ and bounded from below by zero, and thus the convergence of the iteration is guaranteed. The effect of the optimization algorithm will be shown in the next section.

For the mode-independent case, we provide the following proposition.

Proposition 3. For the system in (1) and matrices $F_{i}$, the following statements are equivalent.

(I) There exists $K^{*}$ such that (2) is stochastically stable, and $\left\|\mathbf{F}-K^{*} \mathbf{C}\right\| \leq \epsilon_{1}$, where $\epsilon_{1}>0$ is a sufficiently small scalar.

(II) $x(k+1)=\left(A_{r(k)}+B_{r(k)} F_{r(k)}\right) x(k)$ is stochastically stable and $\left\|\mathbf{F C}^{\perp}\right\| \leq \epsilon_{2}$, where $\epsilon_{2}>0$ is a sufficiently small scalar, where

$$
\mathbf{F}=\left[\begin{array}{llll}
F_{1} & F_{2} & \cdots & F_{s}
\end{array}\right], \quad \mathbf{C}=\left[\begin{array}{llll}
C_{1} & C_{2} & \cdots & C_{s}
\end{array}\right] .
$$

The proof is similar to that of Proposition 2, and thus omitted here for brevity.

\section{Numerical examples}

Example 1. Consider a 2-mode Markovian jump system with the following system matrices

$$
\begin{aligned}
& A_{1}=\left[\begin{array}{ccc}
1.5 & 0 & 2.0 \\
1 & 0 & 0.5 \\
0 & 0.2 & -0.5
\end{array}\right], \quad A_{2}=\left[\begin{array}{ccc}
1.8 & 0.5 & 2.0 \\
-0.2 & 0.1 & 1.0 \\
0.15 & -0.2 & -0.2
\end{array}\right], \\
& B_{1}=\left[\begin{array}{cc}
1.2 & 0 \\
0 & 1.5 \\
1 & 0.2
\end{array}\right], \quad B_{2}=\left[\begin{array}{cc}
0.1 & 0.9 \\
-0.2 & 0.8 \\
0.8 & 0.1
\end{array}\right], \\
& C_{1}=\left[\begin{array}{lll}
1 & 0 & 1
\end{array}\right], \quad C_{2}=\left[\begin{array}{lll}
1 & 1 & -0.5
\end{array}\right],
\end{aligned}
$$

and a transition probability matrix $\Pi=\left[\begin{array}{ll}0.6 & 0.4 \\ 0.7 & 0.3\end{array}\right]$. It can be verified easily that the system is not stochastically stable. Choosing $\alpha=10^{5}$ and setting state-feedback stabilizing matrices solved by the approach proposed in Ji et al. (1991) as initial values, a $\gamma_{l t z}^{(2)}=-0.6242<0$ is obtained after one iteration, and a mode-dependent SOF control law can be computed as $K_{1}=$ $\left[\begin{array}{ll}-0.5270 & -0.5989\end{array}\right]^{T}$ and $K_{2}=\left[\begin{array}{ll}-0.6360 & 0.1013\end{array}\right]^{T}$. Furthermore, by employing Theorem 4 and the corresponding algorithm with the same $\alpha$ and initial values, a mode-independent SOF control law $K=\left[\begin{array}{ll}-0.3882 & -0.4070\end{array}\right]^{T}$ is obtained after one iteration. 
Table 1

Numerical results for Example 3.

\begin{tabular}{|c|c|c|c|c|c|}
\hline & $\beta=2.9$ & $\beta=3.3$ & $\beta=3.7$ & $\beta=3.9$ & $\beta=4.0$ \\
\hline Bara and Boutayeb (2005) with $T_{C 1}$ & $x^{*}$ & $x$ & $\times$ & $\times$ & $x$ \\
\hline Bara and Boutayeb (2005) with $T_{C 2}$ & $\checkmark$ & $x$ & $x$ & $\times$ & $x$ \\
\hline Lee et al. (2006) with $T_{B 1}$ & $\times$ & $x$ & $\times$ & $\times$ & $x$ \\
\hline Lee et al. (2006) with $T_{B 2}$ & $\checkmark$ & $\checkmark$ & $\times$ & $\times$ & $\times$ \\
\hline SA with & $\checkmark$ & $\checkmark$ & $\checkmark$ & $\checkmark$ & $\checkmark$ \\
\hline$\alpha$ (1 iteration) & $9 \times 10^{4}$ & $9 \times 10^{4}$ & $9 \times 10^{4}$ & $1.1 \times 10^{5}$ & $1.11 \times 10^{5}$ \\
\hline and obtained $K$ & {$\left[\begin{array}{l}-0.9456 \\
-0.1164\end{array}\right]$} & {$\left[\begin{array}{l}-1.0607 \\
-0.1852\end{array}\right]$} & {$\left[\begin{array}{c}-1.1842 \\
0.0005\end{array}\right]$} & {$\left[\begin{array}{c}-1.1390 \\
0.1125\end{array}\right]$} & {$\left[\begin{array}{c}-1.2085 \\
0.0898\end{array}\right]$} \\
\hline
\end{tabular}

$\checkmark$ means a controller can be found, while $\times$ means cannot.

Example 2. This example is used to show how to optimize the initial values. Consider a 2-mode Markovian jump system with the following system matrices

$$
\begin{aligned}
& A_{1}=\left[\begin{array}{ccc}
2 & 0.1 & -1 \\
0.9 & 0.2 & 0.5 \\
-0.3 & 0.1 & -0.1
\end{array}\right], \quad A_{2}=\left[\begin{array}{ccc}
1.1 & 0.2 & 1.9 \\
-0.2 & 0 & 1.0 \\
0.1 & 0.2 & 0
\end{array}\right], \\
& B_{1}=\left[\begin{array}{c}
-1 \\
0 \\
0.1
\end{array}\right], \quad B_{2}=\left[\begin{array}{c}
0.1 \\
0 \\
-0.5
\end{array}\right], \\
& C_{1}=\left[\begin{array}{lll}
0.8 & 0.1 & 1
\end{array}\right], \quad C_{2}=\left[\begin{array}{lll}
0 & 1 & 1
\end{array}\right],
\end{aligned}
$$

and a transition probability matrix $\Pi=\left[\begin{array}{ll}0.1 & 0.9 \\ 0.6 & 0.4\end{array}\right]$. We firstly found a set of state-feedback stabilizing matrices by the approach in Ji et al. (1991) as $M_{1}^{(1)}=\left[\begin{array}{lll}2.01 & 0.29 & -1.16\end{array}\right]$ and $M_{2}^{(1)}=$ $\left[\begin{array}{lll}0.80 & 0.53 & 1.22\end{array}\right]$. With these initial values, no solution can be found by Algorithm 1, and thus Algorithm 2 with $F_{i}^{(1)}=M_{i}^{(1)}$ are used to find new initial values. After 5 iterations, a set of new initial values satisfying $\left\|M_{\text {inew }}^{(1)} C_{i}^{\perp}\right\| \leq 1$ is obtained as $M_{1 \text { new }}^{(1)}$ $=\left[\begin{array}{lll}1.91 & 0.21 & 0.85\end{array}\right]$ and $M_{2 \text { new }}^{(1)}=\left[\begin{array}{lll}0.49 & 0.61 & 0.70\end{array}\right]$. Then, using Algorithm 1 with $\alpha=10^{4}$ and the new initial values, a $\gamma_{l t z}^{(2)}=$ -0.5791 is obtained after one iteration, and a mode-dependent SOF control law is computed as $K_{1}=2.6511$ and $K_{2}=0.3863$.

Example 3. When Markovian jumps disappear, the obtained results are also applicable to linear discrete-time systems. Consider the following discrete-time system cited from Lee et al. (2006):

$x(k+1)=\left[\begin{array}{ccc}\beta & 0.3 & 2 \\ 1 & 0 & 1 \\ 0.3 & 0.6 & -0.6\end{array}\right] x(k)+\left[\begin{array}{ll}1 & 0 \\ 0 & 1 \\ 1 & 0\end{array}\right] u(k)$,

$y(k)=\left[\begin{array}{lll}1 & 1 & 0\end{array}\right] x(k)$.

The larger $\beta$ is, the more unstable the system (17) becomes. For comparison, we take into account two recent LMI approaches (Bara \& Boutayeb, 2005; Lee et al., 2006), which both require full rank constraints and coordinate transformation on the system matrix $B$ or $C$. For the approach in Lee et al. (2006), we choose the transformation matrices

$T_{B 1}=\left[\begin{array}{ccc}0.5 & 0 & 0.5 \\ -2 & 1 & 2 \\ -1 & 0 & 1\end{array}\right], \quad T_{B 2}=\left[\begin{array}{ccc}0.5 & 0 & 0.5 \\ 0 & 1 & 0 \\ -0.5 & 0 & 0.5\end{array}\right]$,

such that $T_{B 1} B=T_{B 2} B=\left[\begin{array}{ll}I & 0\end{array}\right]^{T}$. For the approach in Bara and Boutayeb (2005), we choose the transformation matrices

$$
T_{C 1}=\left[\begin{array}{ccc}
1 & 1 & 0 \\
0 & 0.4 & 0.4 \\
1 & 2 & 0
\end{array}\right], \quad T_{C 2}=\left[\begin{array}{ccc}
1 & 1 & 0 \\
-0.5 & 0.4 & 1.4 \\
0 & 0 & 0.2
\end{array}\right],
$$

such that $C T_{C 1}^{-1}=C T_{C 2}^{-1}=\left[\begin{array}{ll}I & 0\end{array}\right]$. For our system augmentation (SA) approach, the initial value $M^{(1)}$ is obtained by directly solving the following LMI

$\left[\begin{array}{cc}-X & (A X+B L)^{T} \\ A X+B L & -X\end{array}\right]<0$

and setting $M^{(1)}=L X^{-1}$.

Table 1 gives the numerical results for different approaches. It can be seen easily that the approaches in Lee et al. (2006) and Bara and Boutayeb (2005) are sensitive to the choice of the transformation matrices. These two approaches may not be applied to Markovian jump systems in a straightforward manner, since the full rank requirements on $B_{i}$ or $C_{i}$ may not be satisfied for each mode, and finding appropriate coordinate transformation matrices may not be an easy task. As for the conservatism, our approach, at least for this example, gains the advantage over theirs as well.

Example 4. In this example, we test different iterative approaches using randomly generated systems. These approaches include the widely used iterative LMI (ILMI) approach (Cao et al., 1998), the cone complement linearization (CCL) approach (El Ghaoui, Oustry, \& AitRami, 1997), and a recently developed Newton-like approach (Orsi, 2005; Orsi, Helmke, \& Moore, 2006) for solving rank constrained inequalities with better performance. For each case of different system dimensions, 500 unstable SOF stabilizable system with a stability degree $\rho$ are randomly generated by following the approach used in de Oliveira and Geromel (1997). The closer of $\rho$ to 1 , the more difficult for the system to be stabilized. For ILMI and SA, the maximal allowed iteration number is set to 200, and the stopping criterion is when the relative change of the objective function value is less than 0.0001. For LMIRank, we use the default settings in the software provided by Orsi (2005).

For SA, $\alpha$ is set to $10^{5}$, and the initial value $M^{(1)}$ is obtained by solving (18) directly. If the iteration fails to find a solution with the initial value, Algorithm 2 will be used to obtain an optimized one. Since the considered models have no uncertainties, we eliminate $H_{1}, H_{2}, G_{1}$, and $G_{2}$ in (14) via the nonconservative projection. For LMIRank, the initial value will be generated and optimized via the default trace heuristic provided in the software. For CCL, the initial value will be generated by the standard approach provided in $\mathrm{El}$ Ghaoui et al. (1997), and if it fails, the following trace minimization procedure suggested in Iwasaki (1999) will be used to optimize the initial value

Minimize trace $(P+X)$, subject to

$\left(C^{\perp}\right)^{T}\left(A^{T} P A-P\right) C^{\perp}<0$,

$\left(\left(B^{T}\right)^{\perp}\right)^{T}\left(A X A^{T}-X\right)\left(B^{T}\right)^{\perp}<0$,

$\left[\begin{array}{ll}P & I \\ I & X\end{array}\right] \geq 0$.

For ILMI, the initial value is generated by the approach provided in Cao et al. (1998). If it fails, the algorithm will be restarted with another possible initial value. 
Table 2

Numerical results for Example 4.

\begin{tabular}{|c|c|c|c|c|c|c|c|c|}
\hline \multirow[t]{2}{*}{$(n, l, m, \rho)$} & \multicolumn{4}{|c|}{ Failures } & \multicolumn{4}{|c|}{ Average CPU time } \\
\hline & ILMI & $\mathrm{CCL}$ & LMIRank & SA & ILMI & $\mathrm{CCL}$ & LMIRank & SA \\
\hline$(3,1,1,0.99)$ & 22 & 3 & 3 & 1 & 0.1831 & 0.3542 & 0.3674 & 0.2304 \\
\hline$(4,3,1,0.66)$ & 19 & 0 & 0 & 0 & 0.2062 & 0.3968 & 0.5458 & 0.6075 \\
\hline$(5,1,2,0.99)$ & 21 & 6 & 1 & 0 & 0.4408 & 0.6792 & 1.0184 & 0.3370 \\
\hline$(6,4,2,0.66)$ & 5 & 0 & 0 & 0 & 0.5823 & 0.7651 & 1.4527 & 0.4412 \\
\hline$(7,1,3,0.99)$ & 21 & 2 & 0 & 0 & 0.8311 & 1.8214 & 1.8078 & 0.8410 \\
\hline$(8,3,4,0.66)$ & 70 & 0 & 0 & 0 & 5.2210 & 1.3887 & 2.9802 & 0.8156 \\
\hline$(9,1,2,0.99)$ & 58 & 2 & 0 & 0 & 4.5650 & 2.3267 & 4.7001 & 1.7624 \\
\hline
\end{tabular}

It follows from Table 2 that, for the "easy" cases, the 4 approaches work well except ILMI for $(8,3,4,0.66)$, whereas, for the "difficult" cases, only SA remains possessing high solvability with a mild increase in CPU time. On the whole, SA (that is, our approach) performs best at least for the tested data. It should be stressed that this rough comparison is NOT an accurate predictor of algorithm performance. For specific problems in practice, it is hard to declare that one approach is definitely better than another, and one ought to make a choice according to different situations.

\section{Conclusions}

The SOF stabilization problem for discrete-time Markovian jump systems has been investigated based on a novel representation of the closed-loop system. By virtue of the representation, a new characterization on stochastic stability of the closed-loop system has been established in terms of matrix inequalities. Necessary and sufficient conditions for modedependent and mode-independent SOF stabilizability have been proposed, and an iteration algorithm has been given for their solution. An optimization to initial values may further improve the solvability. Compared with some existing approaches to SOF synthesis, the proposed one has several advantages that make it specific for Markovian jump systems. Numerical examples are used to illustrate the effectiveness and merit of the theoretical results.

\section{Acknowledgements}

The authors would like to express gratitude to anonymous reviewers for their great efforts to improve the quality of the paper.

\section{References}

Bara, G. I., \& Boutayeb, M. (2005). Static output feedback stabilization with $\mathscr{H}_{\infty}$ performance for linear discrete-time systems. IEEE Transactions on Automatic Control, 50(2), 250-254.

Boyd, S., El Ghaoui, L., Feron, E., \& Balakrishnan, V. (1994). Linear matrix inequalities in system and control theory. Philadelphia: Society for Industrial and Applied Mathematics.

Cao, Y., Lam, J., \& Sun, Y. (1998). Static output feedback stabilization: An ILMI approach. Automatica, 34(12), 1641-1645.

Cao, Y., Sun, Y., \& Mao, W. (1998). A new necessary and sufficient condition for static output feedback stabilizability and comments on: Stabilization via static output feedback. IEEE Transactions on Automatic Control, 43(8), 1110-1111.

Chizeck, H. J., Willsky, A. S., \& Castanon, D. (1986). Discrete-time Markovianjump linear quadratic optimal-control. International Journal of Control, 43(1), 213-231.

Costa, O. L. V., \& dePaulo, W. L. (2007). Indefinite quadratic with linear costs optima control of Markov jump with multiplicative noise systems. Automatica, 43(4), 587-597

Costa, O. L. V., Do Val, J. B. R., \& Geromel, J. C. (1997). A convex programming approach to $\mathscr{H}_{2}$ control of discrete-time Markovian jump linear systems. International Journal of Control, 66(4), 557-579.

Costa, O. L. V., \& Fragoso, M. D. (1993). Stability results for discrete-time linearsystems with Markovian jumping parameters. Journal of Mathematical Analysis and Applications, 179(1), 154-178.

Costa, O. L. V., Fragoso, M. D., \& Marques, R. P. (2005). Discrete-time Markov jump linear systems. London: Springer.

Costa, O. L. V., \& Marques, R. P. (2000). Robust $\mathscr{H}_{2}$-control for discrete-time Markovian jump linear systems. International Journal of Control, 73(1), 11-21.
Costa, O. L. V., \& Marques, R. P. (1998). Mixed $\mathscr{H}_{2} / \mathscr{H}_{\infty}$-control of discrete-time Markovian jump linear systems. IEEE Transactions on Automatic Control, 43(1), 95-100.

de Oliveira, M. C., Bernussou, J., \& Geromel, J. C. (1999). A new discrete-time robust stability condition. Systems \& Control Letters, 37(4), 261-265.

de Oliveira, M. C., \& Geromel, J. C. (1997). Numerical comparison of output feedback design methods. In Proceedings of the 1997 American control conference, June (pp. 72-76) Vol. 1

de Souza, C. E. (2006). Robust stability and stabilization of uncertain discrete-time Markovian jump linear systems. IEEE Transactions on Automatic Control, 51(5), 836-841.

do Val, J. B. R., Geromel, J. C., \& Goncalves, A. P. (2002). The $\mathscr{H}_{2}$-control for jump linear systems: Cluster observations of the Markov state. Automatica, 38(2), 343-349.

Du, C., Xie, L., Teoh, J. N., \& Guo, G. (2005). An improved mixed $\mathscr{H}_{2}$ and $\mathscr{H}_{\infty}$ control design for hard disk drives. IEEE Transactions on Control Systems Technology, $13(5), 832-839$.

El Ghaoui, L., Oustry, F., \& AitRami, M. (1997). A cone complementarity linearization algorithm for static output-feedback and related problems. IEEE Transactions on Automatic Control, 42(8), 1171-1176.

Fujimori, A. (2004). Optimization of static output feedback using substitutive LMI formulation. IEEE Transactions on Automatic Control, 49(6), 995-999.

Gadewadikar, J., Lewis, F. L., Xie, L., Kucera, V., \& Abu-Khalaf, M. (2007). Parameterization of all stabilizing $H_{\infty}$ static state-feedback gains: Application to output-feedback design. Automatica, 43(9), 1597-1604.

Gao, H., Lam, J., Xie, L., \& Wang, C. (2005). New approach to mixed $\mathscr{H}_{2}$ and $\mathcal{H}_{\infty}$ filtering for polytopic discrete-time systems. IEEE Transactions on Signal Processing, 53(8), 3183-3191.

Geromel, J. C., Peres, P. L. D., \& Souza, S. R. (1993). Convex analysis of output feedback structural constraints. In Proceedings of the 32th conference on decision and control, December (pp. 1363-1364). Vol. 2

Huang, M., \& Dey, S. (2007). Stability of Kalman filtering with Markovian packet losses. Automatica, 43(4), 598-607.

Iwasaki, T. (1999). The dual iteration for fixed-order control. IEEE Transactions on Automatic Control, 44(4), 783-788.

Ji, Y., Chizeck, H. J., Feng, X., \& Loparo, K. A. (1991). Stability and control of discrete-time jump linear-systems. Control Theory and Advanced Technology, $7(2), 247-270$.

Karan, M., Shi, P., \& Kaya, C. Y. (2006). Transition probability bounds for the stochastic stability robustness of continuous- and discrete-time Markovian jump linear systems. Automatica, 42(12), 2159-2168.

Lee, J.-W., \& Dullerud, G. E. (2006). Uniform stabilization of discrete-time switched and Markovian jump linear systems. Automatica, 42(2), 205-218.

Lee, K. H., Lee, J. H., \& Kwon, W. H. (2006). Sufficient LMI conditions for $\mathscr{H}_{\infty}$ output feedback stabilization of linear discrete-time systems. IEEE Transactions on Automatic Control, 51(4), 675-680.

Orsi, R. (2005). LMIRank: Software for rank constrained LMI problems. http://users. rsise.anu.edu.au/robert/lmirank/.

Orsi, R., Helmke, U., \& Moore, J. B. (2006). A Newton-like method for solving rank constrained linear matrix inequalities. Automatica, 42(11), 1875-1882.

Seiler, P., \& Sengupta, R. (2003). A bounded real lemma for jump systems. IEEE Transactions on Automatic Control 48(9), 1651-1654.

Shu, Z., Lam, J., \& Xiong, J. (2008). Mixed $\mathscr{H}_{2} / \mathscr{H}_{\infty}$ control of discrete-time Markovian jump systems via static output-feedback controllers. In The 17th IFAC world congress, July (pp. 5909-5914) Vol. 17(1).

Syrmos, V. L., Abdallah, C. T., Dorato, P., \& Grigoriadis, K. (1997). Static output feedback-A survey. Automatica, 33(2), 125-137.

Xiong, J., \& Lam, J. (2007). Stabilization of linear systems over networks with bounded packet loss. Automatica, 43(1), 80-87.

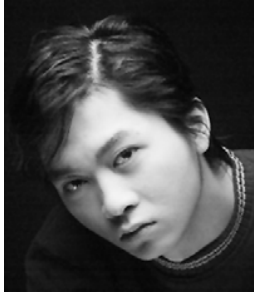

Zhan Shu was born in Nanchang, Jiangxi province, China, in 1982. He received his B.Eng. degree in Automation from Huazhong University of Science and Technology (HUST) in 2003, and the Ph.D. degree in Control Engineering from The University of Hong Kong (HKU) in 2008. He is now a postdoctoral researcher in the Hamilton Institute, National University of Ireland, Maynooth. He is a Member of IEEE. His current research interests include hybrid systems, stochastic systems, positive systems, delay systems, neural networks, robust and adaptive control, estimation and filtering, statistical signal processing, algebraic geometry in system and control theory, systems biology with emphasis on cell regulating networks, immunology, and physiology. 


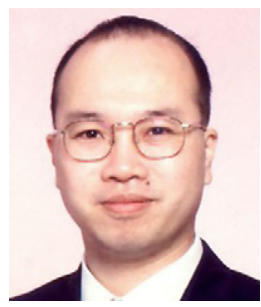

James Lam received a first class BSc degree in Mechanical Engineering from the University of Manchester and was awarded the Ashbury Scholarship, the A.H. Gibson Prize and the $\mathrm{H}$. Wright Baker Prize for his academic performance. From the University of Cambridge, he obtained the M.Phil. and Ph.D. degrees in control engineering. He is a

Scholar and Fellow of the Croucher Foundation. Dr. Lam is now a Professor at the Department of Mechanical Engineering, the University of Hong Kong. Prior to that, he held faculty positions at now the City University of Hong Kong and the University of Melbourne. Professor Lam is a Chartered Mathematician and Chartered Scientist, a Fellow of the Institute of Mathematics and Its Applications, Fellow of the IET, and Senior Member of the IEEE. His research interests are in the analysis and synthesis of dynamic systems which include reduced-order modeling, delay systems, descriptor systems, stochastic systems, multidimensional systems, robust control and filtering. Professor Lam is also actively contributing to journal editorial work. He is Subject Editor of Journal of Sound and Vibration, Associate Editor of Asian Journal of Control, International Journal of Systems Science, International Journal of Applied Mathematics and Computer
Science, IEEE Transactions on Signal Processing, Journal of the Franklin Institute, Dynamics of Continuous, Discrete and Impulsive Systems (Series B: Applications and Algorithms), and Automatica. He is also editorial member of IET Control Theory and Applications, Open Electrical and Electronic Engineering Journal, Research Letters in Signal Processing, International Journal of Systems, Control and Communications, and Multidimensional Systems and Signal Processing.

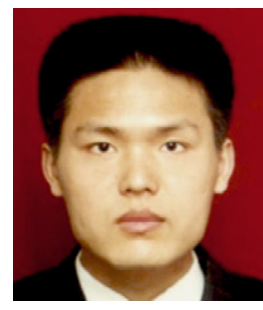

Junlin Xiong earned his B.S. and M.S. degrees from Northeastern University, China, and his Ph.D. degree in Mechanical Engineering from the University of Hong Kong, Hong Kong, in 2000, 2003 and 2007, respectively. Since November 2007, he has carried out his postdoctoral research in School of Engineering and Information Technology, the University of New South Wales at the Australian Defence Force Academy, Australia. His current research interests are in the fields of Markovian jump systems, networked control systems and negative imaginary systems. 